\title{
Magnetic Resonance Imaging in Cervical Facet Dislocation: A Third World Perspective
}

\author{
Manzar Hussain ${ }^{1, a}$, Sadaf Nasir ${ }^{2}$, Ghulam Murtaza ${ }^{1}$, Umber Moeed $^{1}$, Muhammad Ehsan Bari ${ }^{1}$ \\ ${ }^{1}$ Department of Surgery, Aga Khan University Hospital, Karachi, Pakistan \\ ${ }^{2}$ Liaquat National Hospital, Karachi, Pakistan
}

Study Design: Retrospective case series.

Purpose: The objective of our study was to determine the change in management brought about by magnetic resonance imaging (MRI) of the cervical spine in alert and awake patients with facet dislocation and spinal cord injury presenting within 4 hours after injury.

Overview of Literature: Spinal cord injury is a common clinical entity. The role of MRI is well established in evaluating spinal trauma. However, the time at which MRI should be used is still controversial.

Methods: Retrospective data from 2002-2010 was evaluated. All of the alert and awake patients with spinal cord injury, based on clinical examination with facet dislocation diagnosed on lateral cervical spine X-rays, were included. A questionnaire was also conducted, the data of which consisted of demographic details including age and sex, the mechanism of injury, clinical examination, X-ray findings, MRI findings, whether or not surgery was performed and the time elapsed since injury. Data was analyzed using SPSS ver. 17.0. Continuous variables such as age were expressed in terms of mean \pm standard deviation. Categorical variables such as change in management, X-ray/MRI findings and neurological motor level were assessed in terms of percentage.

Results: Fifty patients participated in our study. All these patients had spinal cord injury with defined motor levels. The mean age was $35.5 \pm 8.95$ years (range, 20 to 52 years). Fifty percent showed a motor level at C6 level. None of the patients required any change in management based on the MRI.

Conclusions: MRI of the spine in awake patients within 4 hours after injury does not change the management of patients. However, we can hypothesize that such patients can proceed to traction without waiting for the MRI.

Key Words: Spinal cord injuries, Facet dislocation, Closed reduction, MRI spine

\section{Introduction}

Spinal trauma is frequently seen in tertiary care centers [1]. Management involves rapid diagnosis to identify the cause. Magnetic resonance imaging (MRI) is important in the management of spinal trauma. MRI of the spine not only provides the diagnosis, it may also suggest the possible therapeutic options [2]. Management of the cervical facet dislocation is not subjected to controversies, but the timing of MRI of the spine in cervical trauma is controversial when reduction is considered [3].

Various options are put forward when choosing which procedure should be performed first; either early

Received Mar 2, 2011; Revised Aug 20, 2011; Accepted Sep 9, 2011

Corresponding author: Manzar Hussain, MBBS, FCPS

Department of Surgery, Dow University Hospital, Dow International Medical College, Flat No. a-30, Erum Garden, Block 13/d, Near Hassan Square, Gulshan-e-iqbal, Karachi 75300, Pakistan

Tel: +92-343-2025927, E-mail: Manzary2k@yahoo.com

${ }^{a}$ Present address: Department of Surgery, Dow University Hospital, Dow International Medical College, Karachi, Pakistan 
attempted close reduction or MRI. Those who favor the use of MRI of the spine are of the opinion that the cost of overlooking such injuries is significant and MRI should therefore be performed in every case before the reduction is performed. On the other hand, some surgeons believe that the routine use of MRI of the spine in awake and alert patients before reduction is not warranted because of the clinical observation that patients do not develop any neurological deficits if the reduction is performed first and prompt MRI can be obtained if the patient develops any deficit. In addition, MRI is time consuming and also adds an additional burden on the MRI suite [4]. It must also be remembered that the MRI facility is not available at every center in a third world country such as India. Our study was conducted to evaluate the institutional protocol in India of routinely performing MRI of the spine before closed reduction is attempted.

\section{Materials and Methods}

The study was conducted at the Aga Khan University Hospital (retrospective case series). Retrospective data from 2002-2010 was evaluated. All the alert and awake patients with complete cord injury, based on clinical examination with facet dislocation diagnosed on lateral cervical spine X-rays, were included. No attempt was made to differentiate complete spinal cord injury from spinal shock because of the retrospective nature of the study. Cord contusion was defined as having hyper intensity as seen on T2-weighted images of the cervical spine MRI. Cord compression was defined on the basis of the presence of cerebrospinal fluid (CSF) around the spinal cord on axial T2-weighted MRI images at the concerned level. Cord compression was characterized as mild to moderate in the presence of CSF around the cord and was characterized as severe in the absence of any CSF around the cord. Patients with incomplete cord injury, spinal cord injury without radiological evidence, multiple cervical fractures or minimal or no deficits, were excluded. Those patients who were obtunded were also excluded.

The study was conducted under a waiver of consent since it involved only the collection of data. Retrospective data was reviewed and those patients meeting the inclusion criteria were included. Data was collected on the Performa designed for this purpose. The protocol at our institution has involved obtaining X-ray of the cervical spine in patients presenting with cervical trauma. If the patient is suspected of
Table 1. Magnetic resonance imaging (MRI) $(n=50)$

\begin{tabular}{ll}
\hline \hline MRI findings & $\%$ \\
\hline C6/7 & \\
Herniated disc & 20 \\
Cord contusion & 80 \\
C5/6 & \\
Herniated disc & 30 \\
Cord contusion & 70 \\
C4/5 & \\
Herniated disc & 10 \\
Cord contusion & 90 \\
\hline
\end{tabular}

having spinal cord injury along with facet dislocation, then MRI of the spine is ordered. After the MRI is performed, the patient undergoes closed reduction in an effort to reduce the facet dislocation, which is followed by serial X-rays of the spine. The procedure was fully explained to those patients who were awake and alert before closed reduction was attempted.

Data included in the questionnaire consisted of demographic details including age and sex, mechanism of injury, clinical examination, X-ray findings, MRI findings, whether or not surgery was performed and the time elapsed since injury. All these patients underwent application of cervical traction with the help of Gardner Well's tongs with adjustment according to the level involved for reduction. Data was analyzed using SPSS ver. 17.0 (SPSS Inc., Chicago, IL, USA).

Since the study involves a descriptive case series, continuous variables such as age were expressed in terms of mean \pm standard deviation. Categorical variables such as change in management; X-ray/MRI findings and neurological motor level were assessed in terms of frequency and percentage.

\section{Results}

Fifty patients participated in our study; 45 were males and 5 were females with a mean age of $35.5 \pm 8.95$ years (range, 20 to 52 years). The mechanism of injury was road traffic accident in 40 patients and fall in 10 patients. All these patients showed spinal cord injury with defined motor levels. $50 \%$ of the patients had a C6 motor level, $30 \%$ had a $\mathrm{C} 5$ and $20 \%$ had a C4 level on clinical examination. X-rays of the cervical spine were performed in these patients as part of the trauma protocol. The X-rays showed the facet dislocation at the C6/7 level in $50 \%$ of the patients: $30 \%$ at $\mathrm{C} 5 / 6$ level and 20\% at C4/5 level. MRI findings (Table 1) 
Table 2. Management decision based on MRI $(n=50)$

\begin{tabular}{lc}
\hline \hline Management decision based on MRI & No. of patients \\
\hline Yes & 0 \\
No & 50 \\
\hline
\end{tabular}

MRI: Magnetic resonance imaging.

show the distribution of percentages between cord contusion and herniated disc at each level. Of the 50 patients, 35 showed severe cord compression defined on the basis of the absence of CSF around the cord and 15 showed mild to moderate cord compression. None of the patients required any change in management based on the MRI (Table 2).

\section{Discussion}

The use of MRI of the spine in spinal trauma victims is of paramount importance. It not only helps in diagnosis but also helps in planning possible management options and may help in prognosticating the disease. Closed reduction of the dislocated facets is well established in the management of spinal trauma [5]. The controversy surrounds the timing of the MRI in the case of spinal trauma with facet dislocation.

It is well established in animal studies that the extent of neurologic injury after spinal cord compression is related to both the rate of injury and the duration of compression. It is also known that it is not possible to alter the rate of injury, but the duration of spinal cord compression clearly can be affected by various treatments [6-8]. The time needed to obtain an MRI is often rather long and can be longer when the scanner is being used for elective studies. Additionally, MRI is not available at every center, and a patient may therefore need to be transported to an MRI-equipped facility, risking further injury to an already unstable injury [9]. This aspect is of paramount importance when considering the management of such injuries in patients in 3rd world countries.

On the other hand, the proponents of performing mandatory MRI before attempting closed reduction believe that the force required to cause facet dislocation is sufficient enough to cause damage to the surrounding structures. This has led to the belief that if reduction is attempted before obtaining MRI then the protrusion of the nucleus may go unnoticed. Various investigators have relied on the type of injury, whether complete or incomplete, and the time elapsed since injury to decide whether MRI should be obtained first or whether closed reduction should be attempted first $[6,10,11]$.

Recent case reports of acute neurologic deterioration after the reduction of cervical dislocations in patients with disc herniations have led to the alteration of the treatment algorithm of these injuries at many centers. These reports must be interpreted with caution. Most of the reported cases involve open reduction or reduction under anesthesia rather than awake closed reduction with traction. Eismont et al. [12] reported two cases of neurologic worsening after open reduction of cervical dislocations. One patient received imaging for a disc herniation by intraoperative ultrasound. Although no disc herniation was detected before reduction, cord compression from a herniated disc was found after reduction. The other patient did not receive imaging for a disc herniation before reduction and showed cord compression from a disc herniation demonstrated by myelography after reduction. They also described one patient who showed worsening of upper extremity paralysis while undergoing awake closed reduction with traction. Attempts at closed reduction were then aborted, and the patient recovered to normal after an anterior discectomy and fusion, and posterior reduction and fusion were performed [12]. Similar results have been published by other investigators [13-17]. The results of these studies illustrate that although permanent neurologic worsening has been reported with certain closed-reduction techniques, neurologic worsening seems to be more common after open reduction, usually in the presence of a disc herniation imaged after reduction.

It must be appreciated that closed reduction is performed by gradually increasing the traction, with the aid of cervical spine X-rays. A significant amount of data is available that shows that various authors have attempted closed reduction successfully without any neurological deficits and without the need for any pre-reduction imaging [18,19]. The safety of awake closed reduction with traction, using high weights if necessary, indicates that the distraction across the disc space at the time of reduction somehow limits further injury to the inter vertebral disc and minimizes risk of injury to the spinal cord. Open reduction, closed traction reduction using minimal weight, closed reduction under anesthesia, or spontaneous reduction of dislocated facets may push more disc material posteriorly than if initial axial distraction of the disc space is achieved with higher weight traction. This could account for the higher frequency of reports of neurologic worsening after open reduction, spontaneous closed reduction, or closed reduction by other techniques 
$[20,21]$.

These results suggest that in our cohort of patients obtaining routine MRI of cervical spine before attempting closed reduction did not add significant information in terms of further planning. Most of these patients showed spinal cord contusion along with facet dislocation. In these patients, there is a theoretical chance of improving patient neurological status if prompt action is taken to relieve the pressure by immediate close reduction. It must also be remembered that crucial time is lost while waiting for the MRI. This might be of significant importance in patients with clinical features of spinal cord injury, who present early after the injury and who are diagnosed with facet dislocation. In addition, in a third world country such as India, where the pre hospital care is very rudimentary, precious time is lost. It seems more logical that such patients should be given the maximum chance to improve.

We acknowledge that our study has some inherent weaknesses such as the retrospective nature of the study, the relatively small sample size from single institution absence of long term follow-up and lack of randomization. We also acknowledge that due to the retrospective nature of our study we were not able to differentiate spinal shock from complete spinal cord injury. To the best of our knowledge this is the first publication dealing with this issue coming from a 3rd world country such as India. Our study can serve as a basic source of information for future researchers dealing with this issue. We believe that randomized studies with large sample sizes will help in solving this controversy.

\section{Conclusions}

MRI of the spine in awake patients within 4 hours of injury does not change the management of patients. However, we can hypothesize that such patients can proceed to traction without waiting for the MRI.

\section{REFERENCES}

1. Grauer JN, Vaccaro AR, Beiner JM, et al. Similarities and differences in the treatment of spine trauma between surgical specialties and location of practice. Spine (Phila Pa 1976) 2004;29:685-96.

2. Vaccaro AR, Madigan L, Schweitzer ME, Flanders AE, Hilibrand AS, Albert TJ. Magnetic resonance imaging analysis of soft tissue disruption after flexion-distraction injuries of the subaxial cervical spine. Spine (Phila Pa
1976) 2001;26:1866-72.

3. Vaccaro AR, Falatyn SP, Flanders AE, Balderston RA, Northrup BE, Cotler JM. Magnetic resonance evaluation of the intervertebral disc, spinal ligaments, and spinal cord before and after closed traction reduction of cervical spine dislocations. Spine (Phila Pa 1976) 1999;24:1210-7.

4. Vaccaro AR, Nachwalter RS. Is magnetic resonance imaging indicated before reduction of a unilateral cervical facet dislocation? Spine (Phila Pa 1976) 2002;27:117-8.

5. Cotler JM, Herbison GJ, Nasuti JF, Ditunno JF Jr, An H, Wolff BE. Closed reduction of traumatic cervical spine dislocation using traction weights up to 140 pounds. Spine (Phila Pa 1976) 1993;18:386-90.

6. Carlson GD, Minato Y, Okada A, et al. Early timedependent decompression for spinal cord injury: vascular mechanisms of recovery. J Neurotrauma 1997;14:951-62.

7. Delamarter RB, Sherman J, Carr JB. Pathophysiology of spinal cord injury: recovery after immediate and delayed decompression. J Bone Joint Surg Am 1995;77:1042-9.

8. Anderson GD, Voets C, Ropiak R, et al. Analysis of patient variables affecting neurologic outcome after traumatic cervical facet dislocation. Spine J 2004;4:50612.

9. Grauer JN, Vaccaro AR, Lee JY, et al. The timing and influence of MRI on the management of patients with cervical facet dislocations remains highly variable: a survey of members of the Spine Trauma Study Group. J Spinal Disord Tech 2009;22:96-9.

10. Rizzolo SJ, Piazza MR, Cotler JM, Balderston RA, Schaefer D, Flanders A. Intervertebral disc injury complicating cervical spine trauma. Spine (Phila $\mathrm{Pa}$ 1976) 1991;16(6 Suppl):S187-9.

11. Ng WP, Fehlings MG, Cuddy B, et al. Surgical treatment for acute spinal cord injury study pilot study \#2: evaluation of protocol for decompressive surgery within 8 hours of injury. Neurosurg Focus 1999;6:e3.

12. Eismont FJ, Arena MJ, Green BA. Extrusion of an intervertebral disc associated with traumatic subluxation or dislocation of cervical facets: case report. J Bone Joint Surg Am 1991;73:1555-60.

13. Burke DC, Berryman D. The place of closed manipulation in the management of flexion-rotation dislocations of the cervical spine. J Bone Joint Surg Br 
1971;53:165-82.

14. Doran SE, Papadopoulos SM, Ducker TB, Lillehei KO. Magnetic resonance imaging documentation of coexistent traumatic locked facets of the cervical spine and disc herniation. J Neurosurg 1993;79:341-5.

15. Mahale YJ, Silver JR, Henderson NJ. Neurological complications of the reduction of cervical spine dislocations. J Bone Joint Surg Br 1993;75:403-9.

16. Olerud C, Jónsson H Jr. Compression of the cervical spine cord after reduction of fracture dislocations: report of 2 cases. Acta Orthop Scand 1991;62:599-601.

17. Robertson PA, Ryan MD. Neurological deterioration after reduction of cervical subluxation: mechanical compression by disc tissue. J Bone Joint Surg Br 1992;74:224-7.
18. Cotler HB, Miller LS, DeLucia FA, Cotler JM, Davne SH. Closed reduction of cervical spine dislocations. Clin Orthop Relat Res 1987;(214):185-99.

19. Hadley MN, Fitzpatrick BC, Sonntag VK, Browner CM. Facet fracture-dislocation injuries of the cervical spine. Neurosurgery 1992;30:661-6.

20. Harrington JF, Likavec MJ, Smith AS. Disc herniation in cervical fracture subluxation. Neurosurgery 1991;29:374-9.

21. Rizzolo SJ, Vaccaro AR, Cotler JM, Balderston RA, Flanders AE, Ergener J. Incidence and treatment of disc injury in patients with cervical spine dislocations. In: Annual Meeting of the American Academy of Orthopaedic Surgeons; 1997 Feb 13-17; San Francisco, CA. 